\title{
Indicadores para evaluar la calidad en un curso de capacitación e-learning en México
}

\author{
Alba Alejandra Lira García \\ Profesora de la Facultad de Ciencias de la Conducta de la \\ Universidad Autónoma del Estado de México (Toluca, México) \\ aalirag@uaemex.mx | https://orcid.org/0000-0002-9274-5725
}

Kárilyn Brunett Zarza

Profesora de la Facultad de Ciencias de la Conducta de la Universidad Autónoma del Estado de México (Toluca, México) kbrunettz@uaemex.mx | https://orcid.org/0000-0002-6718-9771

\section{Extracto}

El objetivo general de este artículo es contribuir a la construcción de mejores procesos metodológicos para evaluar la calidad del diseño instruccional, tomando como base el curso e-learning "¿Qué te detiene? De la conciencia individual al empoderamiento colectivo», dirigido a trabajadores del servicio público del Estado de México, el cual se fundamentó en el modelo de diseño instruccional ADDIE: análisis, diseño, desarrollo, implementación y evaluación. La aproximación teórica y metodológica se realiza con base en el análisis cuantitativo y cualitativo de un conjunto de indicadores divididos en dos categorías: valoración interna (eficiencia terminal, reprobación, abandono escolar y rangos de calificación) y valoración externa (basada en una encuesta de satisfacción construida a partir de categorías específicas, entre ellas, cantidad y calidad del contenido, material didáctico y su correspondencia con las actividades, su evaluación y retroalimentación). Se destaca que las instituciones cuentan con reducidos elementos para medir la calidad de los cursos que ofertan, por lo tanto, la evaluación del diseño instruccional se asume como un proceso holístico cuya reflexión deriva en propuestas de formación e-learning con mayor equidad, calidad y pertinencia social.

Palabras clave: evaluación; indicadores; calidad; curso; e-learning.

Fecha de entrada: 28-04-2021 / Fecha de revisión: 15-06-2021 / Fecha de aceptación: 21-06-2021

Cómo citar: Lira García, A. A. y Brunett Zarza, K. (2021). Indicadores para evaluar la calidad en un curso de capacitación e-learning en México. Tecnología, Cienciay Educación, 20, 83-102. https://doi.org/10.51302/tce.2021.610 


\title{
Indicators used to assess the quality of an e-learning course in Mexico
}

\author{
Alba Alejandra Lira García \\ Kárilyn Brunett Zarza
}

\begin{abstract}
The general objective of this article is to contribute to the construction of better methodological processes to evaluate the quality of instructional design. Based on the e-learning course «What is stopping you? From individual conscience to collective empowerment» aimed at public service workers in the State of Mexico. Which was based on the evaluation phase of the ADDIE: analysis, design, development, implementation and evaluation. The theoretical and methodological approach refers to the quantitative and qualitative analysis of a set of indicators divided into two categories: internal assessment (terminal efficiency, failure, school dropout and qualification ranges) and external assessment (based on a satisfaction survey, constructed from specific categories, including quantity and quality of content, teaching materials and their correspondence with activities, their evaluation and feedback). Emphasizing that the institutions have limited elements to measure the quality of the courses they offer. Therefore, the evaluation of the instructional design is assumed as a holistic process whose reflection results in e-learning training proposals with greater equity, quality, and social relevance.
\end{abstract}

Keywords: evaluation; indicators; quality; course; e-learning.

Citation: Lira García, A. A. and BrunettZarza, K. (2021). Indicators used to assess the quality of an e-leaming course in Mexico. Tecnologia, Ciencia y Educación, 20, 83-102. https://doi.org/10.51302/tce.2021.610 


\section{Sumario}

1. Introducción

2. Consideraciones sobre la evaluación de la calidad en el diseño instruccional

3. Evaluación e-learning como proceso holístico

4. Valoración interna: eficiencia terminal, abandono y rango de calificaciones

5. Valoración externa: encuesta de satisfacción y comentarios abiertos

6. Conclusiones

Referencias bibliográficas 


\section{Introducción}

La experiencia de evaluación que se presenta en este estudio de investigación es resultado de la implementación del curso e-learning «¿Qué te detiene? De la conciencia individual al empoderamiento colectivo», el cual tuvo como objetivo «fomentar el empoderamiento de las mujeres en lo individual y lo colectivo". La duración de dicho curso fue de 25 horas, divididas en 5 módulos de 5 horas cada uno. Participaron un total de 107 personas, de las cuales 24 fueron hombres, 81 mujeres y 2 no registraron su género. La característica común entre los participantes fue su desempeño como servidores públicos del Estado de México.

El modelo de diseño instruccional empleado para sustentar metodológicamente la estructura del curso fue el ADDIE, cuyas siglas se adaptan al español estableciendo fases procedimentales: análisis, diseño, desarrollo, implementación y evaluación. La última fase tiene dos finalidades:

- Su reflexión sirve para la toma de decisiones concretas sobre los elementos que se quieren modificar.

- Permite construir indicadores, criterios y comparaciones para valorar la calidad global del curso y la estructura interna de los módulos.

La problemática que se identifica en la literatura especializada sobre evaluación de la calidad del diseño instruccional es que esta varía según el contexto institucional, sus fundamentos conceptuales o el momento en que la valoración se realiza: como elemento diagnóstico, para verificar los resultados y para modificar el proceso instructivo. En México es frecuente que los cursos e-learning de capacitación sean proporcionados por instituciones no vinculadas al ámbito educativo, por lo cual los vacíos metodológicos en el diseño instruccional y sus procedimientos de evaluación son comunes. Con esta experiencia se pretende contribuir al esfuerzo de este tipo de instituciones.

La «capacitación» se define como la educación dirigida a un sector particular que cuenta con una formación inicial y cuyas necesidades de educación continua se han determinado previamente con el objeto de optimizar el desempeño y la eficacia de cada empleado. En este caso, la divulgación del conocimiento con perspectiva de género está indudablemente favorecido por el e-learning, principalmente porque reduce el costo financiero y produce resultados cuantificables en un tiempo relativamente corto. 
Sin embargo, este tipo de iniciativas se han venido impulsando institucionalmente con un amplio desconocimiento sobre los requerimientos mínimos de diseño instruccional y, en consecuencia, los resultados de la evaluación de la calidad se concentran en la evidencia empírica del número final de trabajadores capacitados. Con la propuesta metodológica que se presenta se pretende ampliar cualitativamente los criterios de calidad a partir de la información que aportan los teóricos del diseño instruccional.

\section{Consideraciones sobre la evaluación de la calidad en el diseño instruccional}

En términos de García (2017), los fundamentos teóricos de la educación a distancia no se restringen a un paradigma único, más bien transitan entre las teorías pedagógicas y las teorías psicológicas del aprendizaje, las cuales quedan también intervenidas por la crítica e interpretación sociológica. Se incluye el análisis a partir de la perspectiva de la evaluación educativa. Dichas cuestiones ponen de manifiesto un conjunto de reflexiones que deben ser consideradas a la hora de esbozar un proceso instructivo.

Desde la perspectiva racionalista, la evaluación sirve para alcanzar la máxima eficiencia y eficacia de un sistema instruccional, además de considerar elementos asociados a la cultura y a la política de las instituciones en las que se genera, con miras a justificar la toma de decisiones

Desde la perspectiva racionalista, la evaluación sirve para alcanzar la máxima eficiencia y eficacia de un sistema instruccional y la pertinencia de los cursos. La evaluación se considera como un elemento de control con indicadores medibles y observables, pero "solo en raros casos hay investigadores que examinan las funciones que la evaluación cumple para validar y reproducir ciertas formas de conocimiento» (Moreno, 2016, p. 102).

El tema de la calidad en el diseño instruccional muestra un panorama complejo. En términos más amplios:

Un sistema educativo a distancia es de calidad cuando sus características hacen posible satisfacer las crecientes necesidades sociales de formación que requiere el desarrollo social de la comunidad a la que sirve y al momento en que se aplica (Yee, 2012, p. 105).

Sin embargo, la perspectiva desde la cual se asume que la educación mediada por tecnología es una vía de acceso a la equidad y a la atención a grupos específicos de población no siempre corresponde a la realidad social, a las necesidades específicas y a las oportunidades reales de acceso a internet, recursos tecnológicos y habilidades digitales. De manera que la tecnología, vista como bien social, es cuestionada a partir del «determinismo tecnológico», entendido como «un conjunto de posturas que les adjudican a la técnica y a la 
tecnología efectos sociales previamente establecidos. [...] los efectos de la tecnología son los que moldean al hombre, sus deseos, necesidades y formas de significar el mundo [...] [que] determinan el imaginario social» (Colangelo, 2016, pp. 16-18).

Rubio (2003) indica que los modelos comúnmente empleados en la evaluación e-learning centran su atención en elementos parciales de la estructura de un curso, principalmente considerando el tipo de tecnología empleada, pero haciendo escasa referencia a los elementos pedagógicos que inciden en el logro de los objetivos de aprendizaje, por ello la autora propone la construcción de modelos de evaluación mixto o de enfoque global que tiendan a la valoración cualitativa del logro del aprendizaje y no solo de su estructura.

En México la educación universitaria mediada por tecnología ha adoptado recientemente criterios que tienden a establecer principios y estándares únicos para la evaluación y acreditación de los programas educativos en modalidad a distancia y mixta ${ }^{1}$. Entre ellos destaca lo establecido por los Comités Interinstitucionales para la Evaluación de la Educación Superior (CIEES) de 2017 y 2018. No obstante, para el caso de los cursos de capacitación, esta información es responsabilidad absoluta de las instituciones, así como también lo es la definición de los estándares, de los parámetros y de los instrumentos de verificación.

Según Martínez (2010), «un indicador puede definirse como la medición de un objetivo a perseguir, de un recurso a movilizar, de un efecto alcanzado, de una estimación de calidad, o una variable de contexto» (p. 5). El término «indicador» se relaciona con otros, tales como «variable» o «dimensión». Lo significativo de esta selección, de acuerdo con Martínez, es «precisar el nombre y la definición de cada indicador, y ubicar[lo] en el marco de referencia que se esté utilizando. Habrán de señalarse, además, las fuentes de obtención de la información de base; la fórmula de cálculo y, en su caso, los criterios para ello» (p. 10).

Cuando la evaluación de la calidad de los cursos tiene como finalidad legitimar las acciones institucionales, los resultados se reducen al porcentaje de participantes que logran culminar un proceso formativo; es decir, se evitan las valoraciones externas, que den cuenta del nivel de logro de los objetivos de capacitación. Para ello, Aguaded y Medina-Salguero (2015) señalan la importancia de
Cuando la evaluación de la calidad de los cursos tiene como finalidad legitimar las acciones institucionales, los resultados se reducen al porcentaje de participantes que logran culminar un proceso formativo

1 En este sentido es reconocible la experiencia de diversos organismos internacionales, entre ellos: Instituto Latinoamericano y del Caribe de Calidad en Educación Superior a Distancia (CALED), European Foundation for Quality in E-Learning (EFQUEL) y Canadian Association for Community Education (CACE) (Ávila y Barragán de Anda, 2015). 
considerar el ciclo de satisfacción de las necesidades y expectativas de los destinatarios del e-learning. Ambos autores se refieren a los componentes del estándar UNE-66181 de AENOR (Asociación Española de Normalización y Certificación) cuando indican que «los grados de satisfacción de los usuarios de la formación virtual va[n] en función de la discrepancia, positiva o negativa, entre lo que esperaban (expectativas de inicio) y lo que han recibido» (p.126)

En ese sentido, se coincide con Rubio (2003) en la adopción de procedimientos de evaluación que no pongan el énfasis exclusivamente en los resultados finales, sino también en la valoración de la calidad global del curso, considerando que la población meta es el centro del proceso, además de tomar en cuenta las características institucionales. Los modelos que aportan esta posibilidad son el «modelo sistémico» de Vann Slyke et al., el «modelo de los cinco niveles de evaluación» de Marshall y Shriver (McArdle, 1999) y el «modelo de los cuatro niveles» de Kirkpatrick (1994) (todos ellos citados en Rubio, 2003). La característica común en dichos modelos es el grado en que un curso online permite transferir o aplicar los conocimientos adquiridos al puesto de trabajo.
La característica común del «modelo sistémico» de Vann Slyke et al., del «modelo de los cinco niveles de evaluación» de Marshall y Shriver y del «modelo de los cuatro niveles» de Kirkpatrick es el grado en que un curso online permite transferir o aplicar los conocimientos adquiridos al puesto de trabajo

\section{Evaluación e-learning como proceso holístico}

Si bien en el modelo ADDIE la evaluación es una fase al final del diseño instruccional, esta cumple una función holística de retroalimentar al resto de las fases del proceso (análisis, diseño, desarrollo e implementación). La evaluación queda dividida en tres momentos diferenciados (diagnóstica, de proceso y final), que, en conjunto, son el hilo conductor de todo el proceso de diseño instruccional.

\author{
La evaluación queda dividida \\ en tres momentos diferenciados \\ (diagnóstica, de proceso y final), \\ que, en conjunto, son el hilo \\ conductor de todo el proceso \\ de diseño instruccional
}

Para el caso que se describe, los datos construidos con la evaluación diagnóstica se recopilan en el cuadro 1. Ordenados de mayor a menor, se destaca que los funcionarios públicos desempeñan actividades laborales relacionadas con la atención a las mujeres. Pero este dato no es suficiente. Fue necesario indagar en los objetivos particulares de las instancias de procedencia porque las características del estudiantado que se establecen durante el análisis no son idénticas a las características reales. Es recomendable conocer sus condiciones de trabajo, sus experiencias previas, sus expectativas y posibilidades, pues con ello se estarán ajustando los cursos al perfil de ingreso de los destinatarios (Meza, 2012). 
Cuadro 1. Clasificación por institución de procedencia

\begin{tabular}{l|c|c|}
\multicolumn{1}{c|}{ Dependencia de Gobierno } & Total & Porcentaje \\
\hline Dirección de Bienestar Social para Adultos Mayores & 42 & $39 \%$ \\
\hline Dirección de Bienestar Social para la Mujer & 33 & $31 \%$ \\
\hline Dirección de Asistencia Jurídica y Psicológica & 30 & $28 \%$ \\
\hline Secretaría de Desarrollo Social & 1 & $1 \%$ \\
\hline Vocalía & 1 & $1 \%$ \\
\hline Total & 107 & $100 \%$ \\
\hline
\end{tabular}

Fuente: elaboración propia basada en los datos obtenidos al finalizar el curso.

Las instancias participantes en el curso comparten un objetivo común: promover el bienestar social integral de hombres y mujeres mediante el diseño de mecanismos que contribuyan a disminuir la violencia de género, y mejorar el acceso a las oportunidades para el empoderamiento y atención especializada. Sin embargo, al centrar el diagnóstico en el ámbito institucional, se obtienen pocos datos sobre el tipo de información concreta y necesaria para resolver problemas concernientes a la desigualdad de género desde el ámbito de la atención social. Es decir, pocas veces se indaga en las necesidades reales de capacitación y en las expectativas de sus recursos humanos.

Otros elementos considerados en la evaluación de diagnóstico fueron el tiempo previsto para acceder al curso, el espacio físico disponible, el acceso a internet y la experiencia de uso de plataformas educativas o foros online. Un $78 \%$ de los participantes tenía acceso a una computadora e internet dentro de su horario laboral y un $67 \%$ estaba familiarizado con los cursos de capacitación online.

A partir de la evaluación de diagnóstico se determinó el perfil profesional2: dirigido a personas que trabajan en el servicio público en el Estado de México, preferentemente interesadas e interesados en aplicar temas relacionados con la perspectiva de género para mejorar su desempeño laboral en el ámbito del servicio público.

Como se puede observar, el perfil profesional anterior no especifica con claridad el grado de complejidad del contenido, por lo que el nivel de logro es ambiguo. Puede tratarse

2 El término equivalente en México es «perfil de egreso». Según el Diccionario de americanismos de la Real Academia Española, en muchos países de Hispanoamérica, el término «egreso» significa «finalización del programa de estudios de una carrera de educación superior». 
de la introducción a conceptos o teorías esenciales, del uso del vocabulario inclusivo, o bien de la transferencia de estos al ámbito del trabajo, como, por ejemplo, en el caso de la solución de conflictos.

La imprecisión del nivel de logro puede influir de manera significativa en el abandono del curso, incrementando la posibilidad de que los usuarios anticipen expectativas disímiles de formación y que no se sientan satisfechos con la estructura y contenidos del curso, etc., provocando que la escasa información inicial impida que la población meta pueda seleccionar el curso en función de sus necesidades, intereses y preferencias (Aguaded y MedinaSalguero, 2015).

Por otro lado, la evaluación del diseño instruccional sustenta las fases de diseño y desarrollo al valorar el orden con el que se presentan los contenidos, la estimación del tiempo destinado al aprendizaje y la resolución de las actividades. Es decir, garantiza que la cantidad de actividades, la carga de trabajo y los objetivos particulares se organicen de manera equilibrada. Las lecciones

\section{Las lecciones instruccionales o modulares contienen componentes básicos que deben ser verificados como unidades independientes o elementos que integran un todo} instruccionales o modulares contienen componentes básicos que deben ser verificados como unidades independientes o elementos que integran un todo. Así, por ejemplo, cuando una lección contiene texto, vídeo e interactivos multimedia, se valora de manera independiente la calidad de cada elemento. Para ello, el diseñador instruccional elabora tablas de contenido en las que se suman el tiempo acumulado de estudio y la elaboración de las actividades o tareas de evaluación en relación con el objetivo de aprendizaje. Este procedimiento puede ser denominado, de manera genérica, como la «evaluación del proceso».

Específicamente, el proceso de enseñanza-aprendizaje se despliega durante la implementación. En esta fase es oportuno considerar las diferencias elementales de la evaluación del aprendizaje: formativa y sumativa. En su conjunto, estas proporcionan información sobre el nivel de logro de los estudiantes antes, durante y al finalizar el proceso instructivo, considerando como preguntas fundamentales ¿por qué evaluar?, ¿cómo sistematizar el proceso? y ¿qué hacer con los resultados de la evaluación? La relación entre el diseño instruccional es tan estrecha que su conceptualización hace referencia a la evaluación continua, como es el caso de la siguiente definición:

Un proceso dialéctico, sistémico y flexible, cuyas múltiples fases y componentes de planificación se abordan y se trabajan de forma simultánea para seleccionar adecuadamente, después de un análisis y evaluación, los medios y estrategias de enseñanza, de manera que permitan la construcción y reelaboración de aprendizajes significativos por parte de la población-usuario, en función de los tipos de conocimientos que deberán aprehenderse (Polo, 2003, citado en Ávila y Barragán, 2015, p. 13). 
Al parecer no hay un consenso teórico común para definir la calidad de los cursos e-learning, aunque se reconoce la necesidad de adaptar los métodos tradicionales de evaluación educativa a las necesidades de los cursos a distancia, ampliando las categorías, los indicadores y las variables hacia una esfera de análisis cualitativo. Autores como Moreno (2007) sostienen que «la calidad de la educación a distancia debe ser evaluada con las mismas exigencias que cualquier modalidad, solo que con parámetros adecuados a sus peculiaridades» (p. 30). Por el contrario, otros, como Weller (2013), argumentan que «las medidas de calidad convencionales no son apropiadas para los MOOC [massive online open courses], ya que los objetivos e intenciones tanto de los alumnos como de la institución son diferentes en la educación formal y en los MOOC» (Universidad de Salamanca, 2015, p. 16).

\section{Valoración interna: eficiencia terminal, abandono y rango de calificaciones}

El cuadro 2 muestra los resultados globales cuantitativo del curso. Se han considerado los indicadores tradicionales de educación: «eficiencia terminal», «abandono escolar», «rango de calificaciones», «aprobados», «suspensos» y «calificación promedio». Vistos en su conjunto estos indicadores arrojan poca información específica que pueda emplearse para modificar el diseño instruccional, pero, cuando se analizan en función de su definición estadística y se comparan, es posible apreciar los ajustes en cada uno de los módulos.

\author{
Como se puede observar en \\ el cuadro 2 , en este estudio \\ de investigación se han \\ considerado los indicadores \\ tradicionales de educación: \\ «eficiencia terminal», «abandono \\ escolar», «rango de calificaciones», \\ «aprobados», «suspensos»y \\ «calificación promedio»
}

Cuadro 2. Indicadores sobre los datos finales del curso

\begin{tabular}{c|c|c|c|c}
\hline $\begin{array}{c}\text { Total de } \\
\text { inscritos }\end{array}$ & $\begin{array}{c}\text { Concluyeron } \\
\text { las actividades }\end{array}$ & $\begin{array}{c}\text { Eficiencia } \\
\text { terminal }\end{array}$ & $\begin{array}{c}\text { Abandono } \\
\text { escolar }\end{array}$ & $\begin{array}{c}\text { Calificación } \\
\text { menor }\end{array}$ \\
\hline 107 & 91 & $8,50 \%$ & $15,80 \%$ & 2 \\
\hline $\begin{array}{c}\text { Calificación } \\
\text { mayor }\end{array}$ & $\begin{array}{c}\text { Rango de } \\
\text { calificaciones }\end{array}$ & $\begin{array}{c}\text { Número de } \\
\text { aprobados } \\
(>7 \text { puntos })\end{array}$ & $\begin{array}{c}\text { Número de } \\
\text { suspensos } \\
\text { (<7 puntos) }\end{array}$ & $\begin{array}{c}\text { Calificación } \\
\text { promedio }\end{array}$ \\
\hline 11 & 91 & 68 & 23 & 8,6 \\
\hline
\end{tabular}


La «eficiencia terminal» es una medida de la proporción de estudiantes que logran finalizar cada nivel o tipo educativo respecto del total de quienes iniciaron sus estudios tantos ciclos atrás como lo indique una trayectoria ideal (Instituto Nacional para la Evaluación de la Educación en México [INEE], 2020). En este caso, la trayectoria ideal significó alcanzar 25 horas de trabajo en línea con el menor número de abandonos y de suspensos. El cálculo de la eficiencia terminal «permite conocer la eficacia del sistema en el avance ideal de los alumnos, con lo cual se pueden identificar áreas de mejora en la equidad y eficacia del egreso oportuno» (INEE, 2020).

Ahora bien, ajustando dicho indicador a las características de un curso e-learning, cuando el porcentaje de eficiencia terminal supera el $30 \%$ puede considerarse como satisfactorio (Alemán de la Garza et al. 2012), ya que, en el caso de los MOOC, la eficiencia terminal promedio tiene una tasa de finalización menor al $10 \%$ (Universidad de Salamanca, 2015). Aunque los resultados dependen de las características del público destinatario, en el caso del presente curso, la eficiencia terminal fue del 8,50\%, de modo que los abandonos fueron del 15,80\%. Como se puede observar en el cuadro 3, al desglosar la eficiencia terminal por módulos y considerando variaciones como el tipo de actividad (foro, cuestionario y flujo de cuestionarios), los resultados fueron más bajos en el módulo IV, con un 47,20\%, por lo que se considera que la evaluación y el proceso de enseñanza fueron menos atractivos para los participantes, o bien que la evaluación tuvo poca correspondencia con el contenido de ese módulo.

La "aprobación» se define como el total de alumnos que han acreditado satisfactoriamente las evaluaciones establecidas en los planes y programas de estudio; es decir, es el porcentaje de estudiantes promovidos al siguiente ciclo. «La información proporcionada por este indicador es un elemento importante que interviene [en el] momento de analizar el rendimiento escolar Quitar espacio antes de punto y coma[;] asimismo, permite evaluar la eficiencia del sistema educativo» (Secretaría de Educación Pública [SEP], 2019, p. 22).

El cuestionario del módulo II generó el mayor número de suspensos (7), seguido del módulo IV (5). Es así como se sugiere verificar la pertinencia de las preguntas en relación con el contenido de dicho módulo. Sin embargo, los foros tuvieron 0 suspensos, además de crear vínculos de interacción entre el estudiantado, lo que se traduce en redes de conocimiento. Este aspecto específico del módulo II también habrá de modificarse (véase cuadro 3).

De un total de 108 personas, suspendieron 23. Por lo tanto, más del $80 \%$ de los registrados aprobaron las actividades de evaluación. Este mismo dato, interpretado específicamente por cada módulo, sugiere que no es posible apreciar una diferencia menor al $10 \%$ respecto a los que concluyeron sus actividades, teniendo en cuenta que

La «eficiencia terminal» es una medida de la proporción de estudiantes que logran finalizar cada nivel o tipo educativo respecto del total de quienes iniciaron sus estudios tantos ciclos atrás como lo indique una trayectoria ideal 
la cantidad mayor de suspensos en el módulo II fue de 7 personas. El mismo módulo tuvo el rango de calificaciones más alto $(7,5)$. Esto es la diferencia entre la calificación más baja y la más alta. De acuerdo con el nivel de complejidad de la tarea, los foros tuvieron un valor de $20 \%$ y los cuestionarios del $10 \%$ (véase cuadro 3).

Por «abandono escolar» se entiende el número de alumnos que dejan la escuela de un ciclo escolar a otro, por cada cien que se matricularon al inicio del curso de un mismo nivel educativo. El abandono escolar es un indicador que forma parte de la tríada de indicadores de eficiencia (reprobación, abandono y eficiencia terminal) más representativa del sistema educativo (INEE, 2020).

El abandono escolar general del curso fue del $15 \%$. Sin embargo, considerando las variaciones por módulos, se aprecia que en el módulo III se presentó el mayor porcentaje de abandono: $55,40 \%$ del total de registros iniciales (108). Al finalizar los cinco módulos, 63 personas obtuvieron una calificación positiva. De ellos solo 44 concluyeron todas las actividades. Sobre ese total, se agrega la cifra de participantes que presentaron la prueba de recuperación y que no habían acreditado los cinco módulos, es decir, 5 personas. Todo esto indica la pertinencia de dicho instrumento como recurso para disminuir el abandono e incrementar el número de aprobados, aunque los contenidos o actividades de los módulos III y IV fuesen poco significativos (véase cuadro 3).

La cantidad de personas que participaron en las actividades de cada uno de los módulos es un indicador del tipo de actividad que resultó menos interesante. En concreto, en el flujo de cuestionarios del módulo IV, solo 51 personas concluyeron las actividades en comparación con los 84 participantes (hombre y mujeres) que las concluyeron en el módulo I (véase cuadro 3). Por lo tanto, se propone revisar la evaluación en el módulo IV.

El «foro» es un método que logra unificar los resultados y mantiene constante la participación de la audiencia. Es así como en los foros de los módulos II y IV se encuentran los valores menores en cuanto al rango de calificación, siendo 2,5 y 1,67 respectivamente. Las diferencias más amplias en cuanto al rango de calificación se aprecian en el módulo II $(7,5)$ (véase cuadro 3).

Aunque la sistematización de los datos del cuadro 3 permite hacer un análisis comparativo entre módulos, esto no resultó suficiente. Tomando como referencia a Downes (2013), para el caso de los MOOC, Aguaded y Medina-Salguero (2015) señalan que la suma de las puntuaciones finales determina los resultados para un caso particular de aplicación, pero esto no implica que el proceso formativo haya satisfecho las expectativas de aprendizaje del estudiantado y que los resultados sean similares bajo contextos diversos de aplicación. 
Cuadro 3. Evaluación y valoración interna o por módulos

\begin{tabular}{|c|c|c|c|c|c|c|c|}
\hline & Módulo I & \multicolumn{2}{|c|}{ Módulo II } & Módulo III & Módulo IV & Módulo V & $\begin{array}{l}\text { Examen de } \\
\text { recuperación }\end{array}$ \\
\hline $\begin{array}{c}\text { Tipo de } \\
\text { evaluación }\end{array}$ & $\begin{array}{c}\text { Cuestionario } \\
\qquad(10 \%)\end{array}$ & $\begin{array}{c}\text { Cuestionario } \\
\qquad(10 \%)\end{array}$ & $\begin{array}{l}\text { Foro } \\
(20 \%)\end{array}$ & $\begin{array}{l}\text { Cuestionario } \\
\qquad(15 \%)\end{array}$ & $\begin{array}{c}\text { Flujo de } \\
\text { cuestionarios } \\
(15 \%)\end{array}$ & $\begin{array}{l}\text { Foro } \\
(30 \%)\end{array}$ & $\begin{array}{l}\text { Cuestionario } \\
\text { de } \\
\text { recuperación }\end{array}$ \\
\hline $\begin{array}{l}\text { Total de } \\
\text { inscritos }\end{array}$ & 108 & 108 & 108 & 108 & 108 & 108 & 108 \\
\hline $\begin{array}{c}\text { Concluyeron } \\
\text { las actividades }\end{array}$ & 84 & 80 & 60 & 59 & 51 & 64 & 72 \\
\hline $\begin{array}{l}\text { Eficiencia } \\
\text { terminal }\end{array}$ & $77,70 \%$ & $75 \%$ & $55 \%$ & $54,60 \%$ & $47,20 \%$ & $59 \%$ & $67 \%$ \\
\hline $\begin{array}{l}\text { Abandono } \\
\text { escolar }\end{array}$ & $2,30 \%$ & $25 \%$ & $45 \%$ & $55,40 \%$ & $5,80 \%$ & $41 \%$ & $33 \%$ \\
\hline $\begin{array}{l}\text { Calificación } \\
\text { menor }\end{array}$ & 4 & 2,5 & 7,5 & $\begin{array}{c}5,3 \\
\text { (8 aciertos) }\end{array}$ & $\begin{array}{c}2 \\
\text { (3 aciertos) }\end{array}$ & 8,3 & 5,5 \\
\hline $\begin{array}{l}\text { Calificación } \\
\text { mayor }\end{array}$ & 10 & 10 & 10 & $\begin{array}{c}10 \\
\text { (15 aciertos) }\end{array}$ & $\begin{array}{c}10 \\
\text { (15 aciertos) }\end{array}$ & 10 & 10 \\
\hline $\begin{array}{c}\text { Rango de } \\
\text { calificaciones }\end{array}$ & 6 & 7,5 & 2,5 & 4,7 & 8 & 1,67 & 4,5 \\
\hline $\begin{array}{c}\text { Número de } \\
\text { aprobados con } \\
\text { calificación } \geq 7\end{array}$ & 75 & 73 & 60 & 58 & 46 & 64 & 71 \\
\hline $\begin{array}{c}\text { Número de } \\
\text { suspensos con } \\
\text { calificación < } 7\end{array}$ & 3 & 7 & 0 & 1 & 5 & 0 & 1 \\
\hline $\begin{array}{l}\text { Calificación } \\
\text { promedio }\end{array}$ & 9,3 & 9,3 & 9,7 & $\begin{array}{c}9,4 \\
\text { (14,08 aciertos) }\end{array}$ & $\begin{array}{c}\text { 8,6 } \\
\text { (13 aciertos) }\end{array}$ & 9,9 & 9,6 \\
\hline
\end{tabular}

\section{Valoración externa: encuesta de satisfacción y comentarios abiertos}

En la identificación de los criterios de calidad se considera indispensable optar por aquellos que contribuyan a mejorar la organización del curso de forma holística a partir 
del logro del objetivo general y de la totalidad de los elementos para alcanzarlo con eficiencia y eficacia, evitando el sesgo de un conjunto único de datos: «No hay un grupo meta, sino que cada alumno tiene sus propios objetivos y criterios de éxito, y el éxito del curso dependerá de que cada alumno alcance tales objetivos» (Universidad de Salamanca, 2015, p. 19).

La evaluación de la calidad como modelo global también aparece referenciado como multidimensional, integral o de complejidad. Se trata de procesos orientados a la satisfacción de las necesidades y expectativas de los destinatarios, considerando la construcción de estándares en los que «el nivel de calidad se determinará por la conjunción del nivel de satisfacción asociado a cada indicador» (Rubio, 2003, p. 103).

Para indagar en el grado de satisfacción de los usuarios se elaboró un formulario online en formato de escala Likert y el nivel de satisfacción se subdividió en las categorías «suficiente», «medio» y «bajo». Posteriormente, se asignó una puntuación bruta promedio por cada categoría: 5 a 10 (suficiente), 11 a 15 (medio) y 16 a 25 (bajo). Las dimensiones o rubros de satisfacción fueron los siguientes: objetivos, contenidos, materiales, metodología, actividades y tutorías, como se indica en la primera columna del cuadro 4. Dichas dimensiones atienden a la dimensión pedagógica del diseño instruccional y hacen muy poca referencia a los indicadores técnicos, editoriales y de diseño gráfico. Para Moreno Olivos (2016), la calidad hace referencia a la capacidad institucional de fusionar lo académico y lo tecnológico. Las instituciones, además de gestoras del ambiente de aprendizaje virtual, son las encargadas de verificar el nivel de satisfacción de las expectativas de los usuarios.

El enfoque global de calidad sobrepasa la determinación de los estándares centrados en la estructura y en los contenidos del curso para atender a dos ámbitos diferenciados: la satisfacción de los usuarios y lo referente a los estándares o a los resultados académicos y de aprendizaje obtenidos por los estudiantes, los mismos que, a su vez, «tiene[n] que ver con cuestiones inherentes a la visión, [a la] misión y [a] los valores de las instituciones generadoras de las propuestas académicas» (Muñoz, 2004, citado en Marciniak y Gairin, 2018, p. 219).

Algunas de las dimensiones al respecto son promover la construcción del conocimiento compartido y la interacción dinámica con variaciones en el contenido multimedia e interactivo para facilitar su comprensión. En la escala que se construyó, cada uno de los criterios de satisfacción incluyó un conjunto de cualidades o categorías para valorar la calidad. Por ejemplo, el rubro de actividades incluyó las siguientes afirmaciones:

- La descripción de las actividades que se inician con un verbo permite identificar la acción.

- El grado de complejidad es congruente con el tiempo.

- Hay coherencia entre el tipo de actividad y el contenido temático. 
De acuerdo con la puntuación obtenida, uno de los rubros que había que mejorar fue la tutoría o el acompañamiento a lo largo del curso. La tutoría de las actividades tiene que ver con la inmediatez con la que se pueden resolver las dudas técnicas y académicas, así como con el tiempo o la calidad de la retroalimentación y la notificación de las fechas de entrega. Lo anterior confirma la importancia de la tutoría, aun en los cursos cuyos procedimientos de evaluación son autodirigidos.

\section{Cuadro 4. Puntuación obtenida en la encuesta de satisfacción}

\begin{tabular}{|c|c|c|c|c|}
\hline $\begin{array}{l}\text { Rubro o criterio } \\
\text { de satisfacción }\end{array}$ & $\begin{array}{c}\text { Puntuación } \\
\text { obtenida }\end{array}$ & $\begin{array}{l}\text { Suficiente } \\
\text { (mantener) }\end{array}$ & $\begin{array}{l}\text { Medio } \\
\text { (mejorar) }\end{array}$ & $\begin{array}{c}\text { Bajo } \\
\text { (verificar el } \\
\text { proceso) }\end{array}$ \\
\hline Objetivos & 7,8 & $\begin{array}{l}\text { Puntuación bruta } \\
\text { promedio de } \\
5 \text { a } 10 .\end{array}$ & $\begin{array}{c}\text { Puntuación bruta } \\
\text { promedio de } \\
11 \text { a } 15 .\end{array}$ & $\begin{array}{c}\text { Puntuación bruta } \\
\text { promedio de } \\
16 \text { a } 25 \text {. }\end{array}$ \\
\hline $\begin{array}{l}\text { Contenidos y } \\
\text { materiales }\end{array}$ & 8,1 & $\begin{array}{l}\text { Puntuación bruta } \\
\text { promedio de } \\
5 \text { a } 10 .\end{array}$ & $\begin{array}{c}\text { Puntuación bruta } \\
\text { promedio de } \\
11 \text { a } 15 .\end{array}$ & $\begin{array}{c}\text { Puntuación bruta } \\
\text { promedio de } \\
16 \text { a } 25 \text {. }\end{array}$ \\
\hline Metodología & 7,9 & $\begin{array}{l}\text { Puntuación bruta } \\
\text { promedio de } \\
5 \text { a } 10 .\end{array}$ & $\begin{array}{c}\text { Puntuación bruta } \\
\text { promedio de } \\
11 \text { a } 15 .\end{array}$ & $\begin{array}{c}\text { Puntuación bruta } \\
\text { promedio de } \\
16 \text { a } 25 .\end{array}$ \\
\hline $\begin{array}{l}\text { Actividades y } \\
\text { evaluación }\end{array}$ & 9,5 & $\begin{array}{l}\text { Puntuación bruta } \\
\text { promedio de } \\
5 \text { a } 10 \text {. }\end{array}$ & $\begin{array}{c}\text { Puntuación bruta } \\
\text { promedio de } \\
11 \text { a } 15 .\end{array}$ & $\begin{array}{c}\text { Puntuación bruta } \\
\text { promedio de } \\
16 \text { a } 25\end{array}$ \\
\hline Tutorias & 11,5 & $\begin{array}{l}\text { Puntuación bruta } \\
\text { promedio de } \\
5 \text { a } 10 .\end{array}$ & $\begin{array}{c}\text { Puntuación bruta } \\
\text { promedio de } \\
11 \text { a } 15 .\end{array}$ & $\begin{array}{c}\text { Puntuación bruta } \\
\text { promedio de } \\
16 \text { a } 25 .\end{array}$ \\
\hline $\begin{array}{c}\text { Organización, } \\
\text { gestión y soporte } \\
\text { técnico }\end{array}$ & 6,7 & $\begin{array}{l}\text { Puntuación bruta } \\
\text { promedio de } \\
3 \text { a } 6 .\end{array}$ & $\begin{array}{c}\text { Puntuación bruta } \\
\text { promedio de } \\
7 \text { a } 11 .\end{array}$ & $\begin{array}{c}\text { Puntuación bruta } \\
\text { promedio de } \\
12 \text { a } 15 .\end{array}$ \\
\hline Calidad & 12,3 & $\begin{array}{l}\text { Puntuación bruta } \\
\text { promedio de } \\
8 \text { a } 16 .\end{array}$ & $\begin{array}{c}\text { Puntuación bruta } \\
\text { promedio de } \\
17 \text { a } 24 \text {. }\end{array}$ & $\begin{array}{c}\text { Puntuación bruta } \\
\text { promedio de } \\
25 \text { a } 40 .\end{array}$ \\
\hline
\end{tabular}

Fuente: elaboración propia basada en los datos obtenidos al finalizar el curso.

Como se pudo valorar en la información estadística por módulos del cuadro 3, el diseño instruccional deberá ser modificado con mayor detalle en el módulo IV, específicamente 
en elementos como la cantidad de contenido, la evaluación y la metodología, tal como se aprecia en los siguientes comentarios emitidos por los servidores públicos que participaron en el curso:

- «El módulo IV era confuso, pues en el índice no se incluyeron todos los temas ni las actividades que se iban a tratar. Cuando se abrían, a simple vista, parecían cortos, y uno confiaba en que tardaría poco tiempo en terminarlos, pero, una vez iniciados, llevaban mucho tiempo».

- «El material de consulta del módulo IV, como los vídeos, muchas veces no tenía relación con el tema de empoderamiento. Asimismo, algunos títulos de los temas no me parecieron adecuados».

- «Los cuestionarios del módulo IV no fueron nada constructivos, pues en términos prácticos te pedían memorizar cantidades que, si bien servían para medir la magnitud del problema, no te permitían realizar un análisis que te hiciera pensar y razonar».

Otro elemento relevante que hay que considerar es lo adecuado que resultaron los foros de discusión, pues además de contribuir a la reducción del abandono, también fueron una vía adecuada para la construcción del aprendizaje social, para lo cual, incluso, la audiencia sugirió la ampliación de la bibliografía. Los foros fueron relevantes en este caso porque la mayor parte de la población meta compartía objetivos laborales comunes, de modo que se convirtieron en el medio para unificar las diversas perspectivas que compartían los funcionarios públicos del Estado de México en torno a los temas de género.

Dadas las particularidades de la formación e-learning como recurso para la capacitación laboral, esta no solo debería enfocarse en satisfacer las políticas institucionales, sino también en poner énfasis en los elementos que conciernen al diseño instruccional, verificando la calidad de los materiales, las necesidades del estudiantado y la claridad de los objetivos esperados. Todo ello en el marco de la interacción con la plataforma tecnológica, con la finalidad de favorecer el desarrollo de habilidades para el aprendizaje o para el aprendizaje autodidacta. En el caso de la capacitación para el trabajo, resulta indispensable profundizar aún más en las expectativas iniciales de los participantes, puesto que la información que las instituciones proporcionan no siempre se construye basándose en el análisis de las necesidades prácticas o en la solución de problemas en el ámbito laboral.

Además, los modelos de evaluación de la calidad en entornos laborales ponen énfasis en la inclusión de categoría de trasferencia: «La evaluación de la transferencia consiste en detectar si las competencias adquiridas con la formación se aplican en el entorno de trabajo y si se mantienen a lo largo del tiempo» (Rubio, 2003, p. 103). Es decir, verificar que la instrucción mejore la calidad en el servicio que la instituciones brindan a la sociedad. 
La perspectiva holística de la evaluación conlleva cuestionar en qué medida la capacitación para el trabajo es o no asumida como una tarea inmediata que se ve favorecida por entornos formativos automatizados a las necesidades inmediatas de las instituciones, antes que a criterios pedagógicos e instruccionales, lo cual, desde luego, centra la discusión sobre el modo en que las teorías del aprendizaje se adaptan a los entornos virtuales y en el modo en que las instituciones se favorecen de ello para crear ambientes de aprendizaje constructivista o cognitivista de acuerdo a sus posibilidades de tipo tecnológico, académicas y de gestión de recursos humanos.

\section{Conclusiones}

En México, la valoración de la calidad de los cursos de capacitación no pasa por el mismo examen que en el caso de las universidades. Las organizaciones han escogido la modalidad e-learning como un proceso que disminuye los costos e incrementa el número de empleados que se introducen en ámbitos de cocimiento que se identifican como prioritarios, entre ellos la equidad de género. Resulta interesante que la divulgación se haya sistematizado y gestionado por las instituciones, cuando, en el caso de los MOOC, estos cursos son de acceso libre. Esto ocurre, entre otros motivos, porque la autoformación para el trabajo no se asume como un proceso individual, sino más bien como una responsabilidad de las instituciones públicas. Dichas instituciones no siempre están ligadas al ámbito educativo, por lo tanto, los errores metodológicos son con frecuencia inadvertidos por ellas mismas. Al mantenerse al margen de la consulta especializada, estas instituciones pierden la posibilidad de mejorar sus procesos.

Basándonos en el escrutinio teórico presentado en este estudio de investigación se reconoce que «los MOOC son creados por el mismo personal en las mismas instituciones que los cursos convencionales, y son, a menudo, fragmentos extraídos o revisados a partir de material de cursos ya existentes» (Universidad de Salamanca, 2015, p. 16). La instituciones desempeñan un papel fundamental, puesto que los modelos de calidad recuperan la importancia del contexto institucional a partir de las necesidades y expectativas específicas de su personal.

Según indica Rubio (2003) al referirse a la transferencia como vía cualitativa para la calidad, considerando que la mayor parte de la población participante procede de dependencias específicas del Gobierno del Estado de México, es conveniente que el diseño de los cursos de capacitación contenga procedimientos de interacción que contribuyan a la aplicabilidad de los contenidos dentro del ámbito laboral. 
Por ejemplo, en el caso de la Dirección de Bienestar Social para Adultos Mayores se hace necesario proponer temas que unifiquen las necesidades propias de los adultos con materias de género e igualdad tendentes a la resolución de problemas. Lo anterior es conveniente porque, al atender a una audiencia específica, las probabilidades de abandono serán menores. Al mismo tiempo, la audiencia estará más involucrada en el proceso formativo de la institución en su conjunto y no lo considerará como un proceso individual o aislado. En el caso mexicano es importante agilizar el intercambio de información sobre las experiencias académicas e institucionales.

Es necesario concretar el tipo de actividades planteadas, así como el monitoreo del proceso de solución de las tareas, empleando la resolución de problemas y estableciendo redes de conocimiento, las cuales pueden ser más efectivas entre las personas de una misma dependencia. Es indispensable que el diseño instruccional mantenga la mayor uniformidad posible en cuanto a la cantidad de contenido y actividades. Con ello disminuye el abandono modular. En este caso particular, los foros fueron el procedimiento mejor valorado y se sugirió ampliar y no restringir la bibliografía.

Por otro lado, se considera necesaria la formación de profesionales especializados en habilidades de diseño instruccional y en tecnología educativa que logren comunicar el conocimiento científico de manera efectiva y que trabajen en colaboración con las instituciones públicas. La intervención de las instituciones es fundamental para promover la autoformación para el trabajo, evitando que la evaluación final de los cursos se centre en el logro de los objetivos institucionales en detrimento de las expectativas y necesidades sociales que los trabajadores afrontan en el desempeño de su labor.

Las perspectivas teóricas sobre el tema de la evaluación de la calidad del e-learning no logran integrar de manera unidireccional las necesidades, las variables y los factores que inciden en la determinación de los estándares únicos de verificación, por lo cual es necesario construir modelos mixtos o híbridos, ya que todos los modelos «presentan ciertas debilidades. Una de ellas es la carencia de un consenso en cuanto al número de dimensiones. Hay modelos que proyectan evaluar solo tres dimensiones y otros hasta ocho. Otra debilidad se manifiesta en el diferente significado asignado a las dimensiones. Sus interpretaciones son muy diferentes dependiendo del autor que las formuló y de la metodología empleada para alcanzarlas» (Marciniak y Gairín, 2018, p. 12).

Si bien los criterios para valorar la calidad distan de ser comunes, es posible identificar críticas comunes entre los usuarios de la modalidad e-learning. Dichas críticas aparecen documentadas en las investigaciones recientes sobre las principales plataformas educativas, como en el caso de Medina-Salguero y Aguaded (2014) para MiríadaX. Algunas de las conclusiones apuntan a la sobrecarga de trabajo y a las actividades, resaltando la incoherencia con el tiempo de elaboración, la falta de claridad en la oferta real del contenido de aprendizaje, la escasa precisión en los objetivos de aprendizaje, la escasa interactividad y la imprecisión en los conocimientos previos necesarios. Es frecuente que los cursos de capacitación demuestren escasas posibilidades. 
Lo que parece indispensable en la teoría sobre los modelos de calidad es superar la construcción de datos empíricos centrados en la valoración de los resultados, evitando evaluaciones al proceso estructural de los cursos, puesto que las reflexiones teóricas más recientes incorporan también el análisis de las expectativas de los destinatarios, reafirmando que todo proceso educativo considera a los estudiantes como sujetos activos de cambio social, para lo cual es indispensable la colaboración de instancias de evaluación externa a las instituciones, ampliando cada vez más el sentido de la educación online. Coincidimos con Moreno (2007) en que las instituciones de capacitación deberán «actuar con un espíritu de colaboración que nos permita llegar a acuerdos y a compartir esfuerzos, recursos y conocimientos; tal sería el caso de compartir indicadores de "buenas prácticas" con estándares basados en prácticas de buena calidad que se vayan haciendo comunes» (p. 30).

Finalmente, en el modelo de Van Slyke, Kittner y Belanger y en el de Marcelo, Gago y Marcelo, analizados por Marciniak y Gairín (2018), el contexto y las instituciones son esenciales para valorar dimensiones específicas de calidad, "análisis de necesidades formativas, infraestructura, recursos humanos, situación financiera» (p. 233). En México es común que las instituciones públicas proyecten amplios objetivos de capacitación e-learning con escasos recursos financieros o exiguos recursos humanos. En consecuencia, el diseño instruccional tiende a ser escasamente multidisciplinario. Tareas específicas como el diseño de materiales, el aporte científico de los expertos en contenidos o la capacitación de los tutores online son resueltas por perfiles profesionales no especializados, lo que dificulta la realización de análisis comparativos de calidad porque las instituciones proceden de un modo disímil para valorar la calidad de los cursos que ofertan.

\section{Referencias bibliográficas}

Aguaded, I. y Medina-Salguero, R. (2015). Criterios de calidad para la valoración y gestión de MOOC. RIED. Revista Iberoamericana de Educación a Distancia, 18(2), 119-143. http:// revistas.uned.es/index.php/ried/article/ view/13579

Alemán de la Garza, L., Sancho-Vinuesa, T. y Gómez Zermeño, M. (2012.) Análisis de un curso en línea masivo y abierto (MOOC) con una eficiencia terminal atípica. Revista Internacional de Tecnología, Ciencia y Sociedad, 5(1), 91-101. https://journals.eagora.org/rev TECHNO/article/view/460
Ávila González, C. y Barragán de Anda, A. B. (2015). La calidad en los ambientes virtuales de aprendizaje. Una adaptación de categorías e indicadores para los programas a distancia del contexto mexicano. Revista Científica Internacional, 3(10), 1-21. http://www. interscienceplace.org/isp/index.php/isp/ article/view/382

Colangelo, P. L. (2016). Tecnologías de la comunicación: entre el determinismo y el deslumbramiento. Comunicación, 0(34), 11-22. https://dialnet.unirioja.es/servlet/articulo? codigo $=5619272$ 
García Aretio, L. (2017). Educación a distancia y virtual: calidad, disrupción, aprendizajes adaptativo y móvil. RIED. Revista Iberoamericana de Educación a Distancia, 20(2), 9-25. https://www.redalyc.org/articulo.oa?id $=331453132001$

INEE. (2020). Tasa de eficiencia terminal. https:// www.inee.edu.mx/evaluaciones/panoramaeducativo-de-mexico-isen/at02e-eficien cia-terminal/

Kirkpatrick, D. L. (1999). Evaluación de acciones formativas: Ios cuatro niveles. EPISE-Gestión 2000

Marciniak, R. y Gairín Sallán, J. (2018). Dimensiones de evaluación de calidad de educación virtual: revisión de modelos referentes. RIED. Revista Iberoamericana de Educación a Distancia, 21(1), 217-238. http://revistas. uned.es/index.php/ried/article/view/16182/ 16910

McArdle, G. E. (1999). Training Design and Delivery. American Society for Training and Development.

Martínez Rizo, F. (2010). Los indicadores como herramientas para la evaluación de la calidad de los sistemas educativos. Sinéctica, 35, 1-17. http://www.scielo.org.mx/scielo. php? script=sci_arttext\&pid=S1665109X2010000200004

Medina-Salguero, R. y Aguaded, I. (2014). Los MOOC en la plataforma educativa MiríadaX. Profesorado. Revista de Currículum y Formación de Profesorado, 18(1), 137-153. https:// www.redalyc.org/pdf/567/56730662009.pdf

Meza, J. (2012). Modelo pedagógico para proyectos de formación virtual. GIZ. http://www. facico-uaemex.mx/diplomado/2.3\%20 BB\%2OMEZA\%20JOHANA.pdf

Moreno Castañeda, M. (2007). La calidad de la educación a distancia en ambientes virtuales. Apertura, O(6), 19-31. http://www.udgvirtual. udg.mx/apertura/index.php/apertura/article/ view/1215
Moreno Olivos, T. (2016). Evaluación del aprendizaje y para el aprendizaje. Reinventar la evaluación en el aula. Universidad Autónoma Metropolitana.

Polo, M. (2003). Aproximación a un modelo de diseño: ADITE. Revista Docencia Universitaria, 4(1), 68. http://saber.ucv.ve/ojs/index. php/rev_docu/article/view/4549

Rubio, M. ${ }^{a}$ J. (2003). Enfoques y modelos de evaluación del e-learning. RELIEVE. Revista Electrónica de Investigación y Evaluación Educativa, 9(2), 101-120. https://ojs.uv.es/ index.php/RELIEVE/article/view/4332

SEP. (2019). Lineamientos para la formulación de indicadores educativos, 1-94. http://fs.pla neacion.sep.gob.mx/estadistica_e_indica dores/lineamientos_formulacion_de_indicado res.pdf

Unidad Politécnica para la Educación Virtual. IPN. (2020). Criterios para la evaluación técnicopedagógica de recursos didáctico-digitales. https://www.ipn.mx/assets/files/dev/docs/ Asesoria\%20RDD/04CriteriosETPdeR DD.pdf

Universidad de Salamanca. (2015). Calidad y evaluación de MOOC. https://elengua.usal.es/ wp-content/uploads/2016/12/Informe-Cali dad-MOOC.pdf

Van Slyke, C. Kittner, M. y Belanger, F. (1998). Identifying Candidates for Distance education: A Telecommuting Perspective. Proceedings of the America's Conference on Information Systems (pp. 666-668). Baltimore.

Yee Seuret, M.. (2012). La educación a distancia como un bien público: una visión. En M. Moreno Castañeda (Coord.), Veinte visiones de la educación a distancia (pp. 95-110). Universidad de Guadalajara. https://www. researchgate.net/publication/235799306_ Veinte_visiones_de_la_educacion_a_distancia 